\title{
Safe Overprovisioning: Using Power Limits to Increase Aggregate Throughput *
}

\author{
Mark E. Femal and Vincent W. Freeh \\ Department of Computer Science \\ North Carolina State University \\ $\{$ mefemal, vwfreeh\}@ncsu.edu
}

\begin{abstract}
Management of power in data centers is driven by the need to not exceed circuit capacity. The methods employed in the oversight of these power circuits are typically static and ad-hoc. New power-scalable system components allow for dynamically controlling power consumption with an accompanying effect on performance. Because the incremental performance gain from operating in a higher performance state is less than the increase in power, it is possible to overprovision the hardware infrastructure to increase throughput and yet still remain below an aggregate power limit. In overprovisioning, if each component operates at maximum power the limit would be exceeded with disastrous results. However, safe overprovisioning regulates power consumption locally to meet the global power budget. Host-based and network-centric models are proposed to monitor and coordinate the distribution of power with the fundamental goal of increasing throughput. This research work presents the advantages of overprovisioning and describes a general framework and an initial prototype. Initial results with a synthetic benchmark indicate throughput increases of nearly $6 \%$ from a staticly assigned, power managed environment and over $30 \%$ from an unmanaged environment.
\end{abstract}

Keywords: overprovisioning, managing power limits

\section{Introduction}

Our primary motivation is to increase throughput, given defined power limits, by increasing parrallelism. High performance clusters such as BlueGene/L [1] make use of low-power, modest clock rate processors to provide more efficient performance with respect to energy consumption. A similar approach can be taken with frequency scalable CPUs and general-purpose hardware. The CPU is a dominant power consumer in most servers and is consequently our initial focus. In addition, there is a manufacturer commitment towards CPU power conservation as exhibited in the ACPI Specification [2]. In general, scaling the the processor from higher to lower power gears tends to slow down power usage in other devices. The relationship of the CPU frequency $(\mathrm{F})$ and voltage $(\mathrm{V})$ is given by [3]:

$$
C P U \text { Power }=A \cdot C \cdot V^{2} \cdot F
$$

\footnotetext{
* This research was supported in part by an IBM UPP award.
} 
Where $A$ is the activity factor for how frequently gates switch and $C$ is the total capacitance at the gate outputs. Frequency is proportional to voltage; therefore, CPU power is proportional to $F^{3}$. However, to the first approximation, performance is proportional to $F$.

Many large data centers have a goal of managing instantaneous power consumption. The physical infrastructure of data centers is typically partitioned into a set of circuits. These circuits, allocated per rack, provide a maximium quantity of instantaneous power. Data center personnel approach the problem of assigning equipment to racks (provisioning) conservatively. Exceeding the circuit power limit can cause a disruption in service. Therefore, managing instaneous power consumption is often a higher priority than reducing energy consumption.

Complicating the delicate balance of maintaining a safe upper bound on power consumption, significant variation occurs based on the state of connected equipment. A server that boots needs near its maximum rated amount of power. In contrast, lightly loaded and idle servers draw significantly less power. An analysis of work performed and power consumed facilitates safe overprovisioning. This benefit offers data centers the ability to maintain peak performance in defined power limits.

The general concept of overprovisioning is not new in industry. For instance, equipment exists to establish a sequence of power up when disruptions occur along with the ability to monitor aggregate power usage [4]. Hardware provides a means of reacting to power failures and monitoring circuit health; however, it is not a good mechanism for controlling power in a concerted fashion. Such intelligence must be used in environments that suffer from the inability to expand their power infrastructure.

We regard saving energy as a secondary goal in intelligent power allocation. In addition, we choose to not require changes to existing applications. We do not preclude possible gains by allowing applications more control in the decision process but believe solutions that require this to function are too restrictive. Our initial implementation results reflect at least a $6 \%$ gain in throughput in a synthetic benchmark while still remaining below a fixed aggregate power limit. This performance gain includes a static analysis done to ensure cluster nodes are operating at the best gear. In unmanaged environments, using the same benchmark, our implementation provides a throughput gain in excess of $30 \%$.

In section 2 we provide an overview of the principles and design aspects of our model. Section 3 discusses the implementation. Section 4 illustrates preliminary results. Section 5 presents related work and section 6 outlines conclusions and future efforts.

\section{Overview}

It is useful to first formalize the motivations mentioned previously. Given a defined power limit $P_{\text {global }}$, a finite number of nodes can complete work subject to this constraint. If we denote $P_{\text {gear }}$ as the power consumption of a node in a given performance gear, the number of total nodes (all nodes execute in the same gear), can be represented as $N_{\text {gear }}=\left\lfloor P_{\text {global }} / P_{\text {gear }}\right\rfloor$. Next, given the throughput for a node in a given gear represented by the function $T\left(P_{\text {gear }}\right)$, total throughput of all nodes is simply $T_{\text {total }}=T\left(P_{\text {gear }}\right) \cdot N_{\text {gear }}$. The energy efficiency per node, i.e., the useful work 


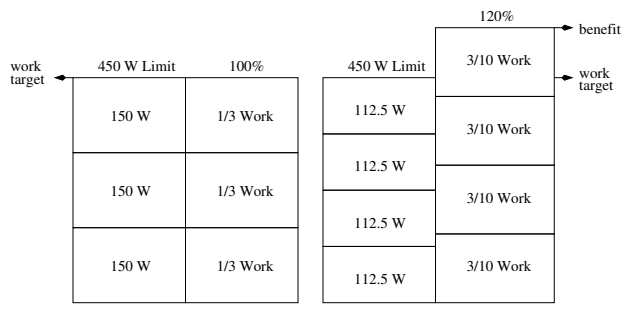

Fig. 1. Managing power to a 450 watt limit based on a $10 \%$ reduction in original work and $25 \%$ reduction in node power.

per unit energy over time $t$ is, $E_{\text {node }}=T\left(P_{\text {gear }}\right) /\left(P_{\text {gear }} \cdot t\right)$. The overall efficiency of $n$ nodes is $E_{\text {total }}=T_{\text {total }} /\left(n \cdot P_{\text {gear }} \cdot t\right)$. A known relationship between $P_{\text {gear }}$ and $T\left(P_{\text {gear }}\right)$, provides the impetus for increasing overall throughput. Modeling theoretical power usage with $P_{\text {gear }}$ is simplified above for clarity. In practice, with varying workloads the maximum, minimum, and average power usage can differ significantly. In addition to finding $N_{\text {gear }}$, it is possible for a mixed set of $P_{\text {gear }}$ values to maximize $P_{\text {global }}$. In general, there is a work benefit when $\Delta P_{\text {global }}>\Delta T_{\text {total }}$. If $P_{\text {global }} \alpha F^{\kappa}$, when $\kappa \leq 1$ and $n$ increases there is a gain in $E_{\text {total }}$.

Figure 1 depicts the aforementioned general strategy. In a given interval, the total power available to all nodes is constrained by a 450 Watt limit. Each node on the left executes unconstrained and consumes 150 Watts while completing $1 / 3$ of the work. In contrast, the overprovisioned nodes on the right are now restricted to $1 / 4$ of the power budget so consume $25 \%$ less power per node. If the restricted nodes execute with a $10 \%$ penalty in work contribution, a reasonable expectation, these nodes provide a realized benefit of $20 \%$ due to exceeding the original work target. This example is presented for illustrative purposes and it provides the incentive to manage power consumption to increase throughput.

\subsection{Local Power Limit}

The locally assigned power limit for each participant in the network is regarded as a mutual decision based on global constraints. Given the global budget, each node is responsible for the suballocation of power at a fine-grained level. Each node operates within its derived maximum power limit. It is free to choose where to set its own target usage level based on need, priority, or other relevant measures.

With this allocation in mind, it is possible for the global power allocation mechanism to account for each node's power need based on the relative difference between its target usage and current limit. This allows flexible policies to be deployed at the local level but still utilizes the same simple interface for the allocation of the global budget. The global budget can be considered to be dynamically assigned based on intelligent devices on the network. For instance, smart racks or uninterruptible power supplies may supply the information for autonomic operation.

At the node's architectural level, each device in a server has an intelligent interface to relay or provide information related to system power draw, available gears, as 


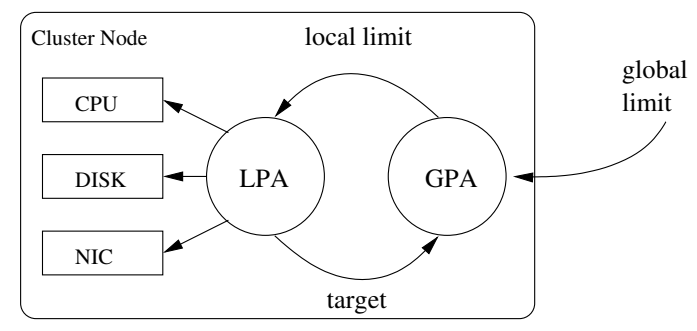

Fig. 2. Relationship of the GPA, LPA, and power-managed devices.

well as minimum and maximum power requirements directly to power management algorithms. Although elements of this are available in laptop systems using the ACPI specification [2], future development of consistent interfaces to hardware should help promote similar access to server sensors using the same specification.

\subsection{Design}

Our general design approach to power distribution is encapsulated with the producerconsumer model. The power producers are regarded as the suppliers of power to various components. The producer at the cluster node level, such as its power supply, may in fact be a consumer at the global level but the approach is conveniently generalized in a hierarchical manner. Thus, each device in the overall system fits in this hierarchy. The policies for each device must be flexible enough to adapt to the unique demands for services from that device. The interface between devices is through its budget and aggregated at upper levels as applicable. The units of budget allocation align with measurement methodology (watts).

\subsection{Components}

A number of logical components comprise the framework and are shown in Figure 2. Starting at the lowest level, device controllers provide the intelligence to determine the performance gear used. Each server node has this functionality aggregated into a cohesive entity referred to as the Local Power Agent (LPA). It is responsible for determining the average power consumption target. There is loosely coupled communication between the LPA and the devices it manipulates with the device controller. A logical Power Message Bus (PMB), implemented as a message queue, facilitates communication between device drivers and the LPA. This flexibility allows for direct control by the LPA as well as the potential for negotiation of power between device drivers.

Another major component of the system is the Global Power Agent (GPA). The GPA is responsible for the coordination and interchange of related messages between nodes. It analyzes messages from the network and makes the appropriate requests to the LPA using the PMB. This assignment is possible due to all participants in a Power Management Group (PMG) broadcasting relevant information to all nodes in the cluster. There is not a one-to-one correspondence between a PMG and a subnet; however, 
Table 1. AMD64 3000+ CPU and system idle power consumption.

\begin{tabular}{|c|c|c|c|c|}
\hline Frequency (Mhz) & BIOS Voltage & CPU (Watts) & New Voltage & System Idle (Watts) \\
\hline \hline 2000 & 1.5 & 89 & 1.5 & 89 \\
\hline 1800 & 1.4 & 66 & 1.4 & 86 \\
\hline 1600 & - & - & 1.35 & 84 \\
\hline 1400 & - & - & 1.3 & 83 \\
\hline 1200 & - & - & 1.2 & 81 \\
\hline 1000 & 1.1 & 22 & 1.1 & 79 \\
\hline 800 & - & - & 1.0 & 77 \\
\hline
\end{tabular}

our initial implementation limits servers in a PMG to be on the same broadcast network. In addition to receiving PMG broadcasts, the GPA responds to other network requests using the Power Exchange Protocol (PEP). These messages include administrative control requests (i.e., setting the global power limit) in our initial prototype.

\subsection{Architecture}

For the initial development and model analysis, a cluster of servers was built using frequency scaling processors. Ten nodes were assembled using the following hardware: 40 GB Maxtor EIDE 7200 RPM disk drives, ASUS K8V motherboards (on-board $1 \mathrm{~Gb}$ NIC), 1 GB of PC3200 DDR SDRAM, and an AMD64 3000+ CPU. All nodes were interconnected on a dedicated $100 \mathrm{Mb}$ switch. Although we regard power distribution as a general allocation problem across a diverse set of hardware architectures and computational resources, our initial implementation is limited due to budgetary reasons.

The entire cluster uses the Linux 2.6 kernel. For frequency and voltage scaling, the AMD PowerNow cpufreq module is used. Modifications were done to augment the ACPI device tables in the BIOS. These additional performance gears were subsequently used in the cpufreq driver as indicated in Table 1. Measured system idle power consumption is shown along with expected CPU power usage obtained from [5]. System idle power reflects reduced power usage from using the HLT instruction. The modified frequency and voltage settings were used to expose additional gears for the purpose of our evaluation.

For calculating system power measurements, two digital multimeters (DMMs) were connected to serial ports on a non-cluster server. Custom software was created to interface with the DMMs located on this host. One meter was configured to measure AC voltage and the other inserted in a serial fashion to measure amperes on a single node. To allow a cluster node to have a notion of power usage, a TCP-based request server was created to allow a node to query power usage as needed. The overhead of this network access is relatively small and a mechanism such as this is needed due to the lack of hardware sensors on each node. In the future, we plan to augment this framework using a more robust collection mechanism for independent cluster node measurement. Our initial research focus is currently on intra-node decisions so the power measurement method is not a limiting factor. Single node measurements are used to extrapolate expected system power usage for multiple nodes. 


\section{Implementation}

As previously discussed in Section 2.3, there are two primary components in our implementation for managing power. The GPA assigns the local power limit based on the aggregate circuit capacity and information received from all cluster nodes. The LPA is responsible for ensuring the target power usage of an individual node is met. Each of these is a separate daemon process and both are implemented as non-priviledged processes.

\subsection{Local Power Agent}

The LPA is the aggregated power control framework for all devices in a cluster node. It is responsible for maintaining a power target and listening for inbound communication on the PMB. Each device has its inherent power characteristics coordinated with other devices by this agent. The PMB provides an abstraction between the idiosyncrasies inherent in each device and the general controller routine. Each device has several gears the LPA manipulates to control overall system power using a device specific routine exposed to the controller.

To simplify the interface to device gears, we regard the lowest power usage gear as the highest numbered performance gear. Thus, performance gear 0 in the state array for each device is the highest power usage gear. Although this is our internal convention, we still refer to increasing the performance gear as an increase in the power usage gear.

The primary focus of the LPA is determining the target power based on the limit assigned by the GPA. This derivation remains flexible to have different policies implemented depending on the behavior desired. Two sample policies include one based on overall load and another might be to optimize for a performance delay characteristic. This policy is not restricted to a single rule, a combination of rules could certainly be employed.

A feedback controller manipulates device gears to meet the target system power set by the policy. To maintain the local power limit, the controller employs a predictor to determine the expected usage in the next epoch. We regard the power limit as an upper bound on instantaneous power usage. A sampling window of size $w$ facilitates keeping system power close to the target. In the initial implementation, the window size is 30 seconds. To prevent excessive gear switching and allow stabilization, a minimum time between changes is enforced. This delay also helps manage the differing capabilities of devices and their subsequent ability to transition to different gears in a specified time interval.

The core controller uses a PID algorithm [6]. If system power is denoted as $S$ and the average power target is $\mu$, the error is $\epsilon=\mu-S$. Next, the controller calculates joules used in the epoch using $j_{e}=t_{e} \cdot \epsilon$. With instantaneous error known and gain constants $G_{1}, G_{2}$, and $G_{3}$, the energy surplus or deficit is

$$
\eta_{e}=G_{1} \cdot j_{e}+G_{2} \cdot \int_{0}^{w} j_{e} d t+G_{3} \cdot\left(j_{e}-j_{e-1}\right) .
$$

With $\eta_{e}$ known, a prediction for the next epoch is determined to ensure the node power limit is not exceeded. For this prediction $\delta$, we find $\delta=\max \left(j_{e}-j_{e-1}, \delta\right)$. 
A non-uniform distribution of $\delta$ values exist for each performance gear. Thus, a table is managed at runtime that represents the quantization of discrete power step values at a resolution defined at compile time for each gear. It is pessimistic due to using the highest value seen in subsequent predictions. The estimator $P_{e}$ for the expected power usage in the next epoch is then found using

$$
P_{e}=S+\frac{\delta+\left(\left(j_{e}-j_{e-1}\right)-\left(j_{e-1}-j_{e-2}\right)\right)}{t_{e}} .
$$

The LPA does not have a strict power limit. Instead, a burstable region exists above and below the GPA assigned limit. The impact to the feedback controller is that if given a burst allocation of $\tau$ and limit of $\omega$, it is $\tau+\omega$ that is regarded as the true limit. The burst allocation allows nodes to respond more rapidly to workload demands in lower performance gears. The current implementation regards the burstable region above the power limit as a soft limit so $P_{e}$ is compared to $\tau+\omega$ to determine if an immediate gear reduction is needed. If no immediate correction is necessary, $\eta_{e}$ is checked against a threshold preset on LPA startup. If the threshold is exceeded and $\eta_{e}<0$ (implies overusage) the CPU gear is decreased. The inverse condition of $\eta_{e}>0$ increases the gear.

\subsection{Global Power Agent}

The GPA's responsibility is to allocate the node power limit based on state information received from all nodes in the PMG. For reliability and scalability, each individual node is responsible for determining the power limit. Although there is explicit trust in ensuring all nodes are well-behaved, this precondition should be acceptable in many environments. All nodes in the PMG are synchronized by periodic UDP broadcasts. Nodes are added or removed from the subnet with corresponding changes done to power limits per the specific policy engine implementation.

Power Management Groups The cluster management policy is shared on all nodes in the current implementation. To allow for multiple logical assignments and allocations on the same broadcast subnet, a cluster identifier is configured for each GPA on daemon startup and is referred to as the PMG. The cluster data structure is an AVL tree, so tree operations are bounded by $O(\lg n)$ where $n$ is the number of PMG nodes (nonmember broadcasts are simply ignored). A dedicated thread is responsible for receiving UDP packets describing the state of other nodes in the PMG as well as reacting to administrative commands (further explained below). This thread uses select() with a timeout to prune the tree based on the time stamp of the last broadcast received for a cluster node and a predetermined maximum broadcast retention value. Within the current implementation, this value is 30 seconds and the broadcast rate is once per second.

Broadcast Messages There are two types of broadcast messages currently sent to participants in a PMG. First, broadcast data packets are sent containing a node's current 
power usage, limit, burst, and target values. In addition, a sequence number is sent that all cluster members use to ensure decisions are computed in a coordinated fashion. This sequence number is initially generated by the first node on a subnet.

In addition to data packets from other members in the PMG, administrative messages can be broadcast to all nodes. Such notifications consist of modifications to the overall power limit to be used by the policy engine of the GPA as well as for setting an immediate administrative node limit used by a support tool. Membership in a PMG is further refined to be either active or passive. In passive mode, broadcasts are sent and received as normal, but inbound administrative messages are ignored. In active mode, the node responds to administrative messages.

\subsection{Support Tools}

Although the long-term intent of the power management framework is to be totally autonomic in operation, the current implementation receives instructions from support tools. Tied into the same communicative protocol as the GPA, a console application monitors the state of either a specific PMG or all nodes running on a given subnet. For administrative control of a given node, a tool exists to interface directly with the LPA (as does the GPA) through shared memory or by sending messages on the PMB. For remote requests, the tool communicates indirectly through the remote node's GPA.

\section{Results}

We evaluated the initial prototype and model on the architecture previously mentioned in Section 2.4. To examine the nature of the tradeoff between throughput and power, we first constructed a series of synthetic CGI [7] programs runnable by an Apache 2.0 web server. We used httperf [8] to generate a sustained workload by using four cluster nodes configured as request clients. A single server was setup to handle requests and clients were configured to overload the server based on a request timeout of five seconds. When the aggregate number of errors for all clients exceeded five percent, we considered the throughput for the server to be maximized for a given performance gear. For the time alloted for a benchmark ( 30 seconds), the average system power consumption was calculated using the multimeters. Due to the high number of nodes needed to generate the workload effectively and current limitations measuring system power, extrapolations based on single node measurements are used to show how effective the solution performs when increasing the number of nodes. An additional cluster node is also used to control the request clients and collect the measured values for reporting purposes.

Our results notation depicts the highest performance gear (and highest power consumption gear) as zero. A consistent theme emerges from the synthesized benchmarks. The highest performing gear does not have the highest performance (i.e., throughput) per unit power. To illustrate this difference in power with respect to throughput, consider Figure 3. It shows the resultant increase in power from gear 1 to 0 is approximately $12.57 \%$; however, the throughput gain is only $6.25 \%$. In contrast, the increase in throughput from gear 6 to 5 is $25 \%$ with only a $6.19 \%$ increase in average power usage. 


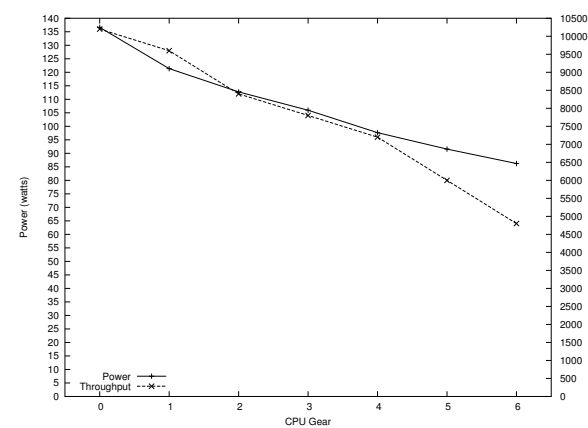

Fig. 3. Merge sort power vs. performance tradeoff.

Table 3. Throughput gains with a 600 watt Table 4. Power and throughput details for inserpower limit and static gear assignment.

\begin{tabular}{|c|r|r|c|c|}
\hline Nodes & Gear & Throughput & Power & Gain \\
\hline \hline 4 & 0 & 40800 & 546.52 & \\
\hline 4 & 1 & 38400 & 485.48 & $-0.06 \%$ \\
\hline 5 & 2 & 42000 & 563.70 & $0.03 \%$ \\
\hline 6 & 3 & 46800 & 529.75 & $12.82 \%$ \\
\hline 6 & 4 & 43200 & 585.96 & $5.56 \%$ \\
\hline 6 & 5 & 36000 & 549.36 & $-11.76 \%$ \\
\hline 6 & 6 & 28800 & 517.32 & $-41.67 \%$ \\
\hline
\end{tabular}

Table 2. Power and throughput details for merge sort.

\begin{tabular}{|c|r|r|r|r|}
\hline Gear & Throughput & Change & Power & Change \\
\hline \hline 0 & 10200 & $6.25 \%$ & 136.63 & $12.57 \%$ \\
\hline 1 & 9600 & $14.29 \%$ & 121.37 & $7.65 \%$ \\
\hline 2 & 8400 & $7.69 \%$ & 112.74 & $6.41 \%$ \\
\hline 3 & 7800 & $8.33 \%$ & 105.95 & $8.49 \%$ \\
\hline 4 & 7200 & $20.00 \%$ & 97.66 & $6.66 \%$ \\
\hline 5 & 6000 & $25.00 \%$ & 91.56 & $6.19 \%$ \\
\hline 6 & 4800 & & 86.22 & \\
\hline
\end{tabular}

Table 2 depicts the raw data collected in this benchmark. In an environment such as this, it is possible to reduce the CPU performance gear to conserve power and increase the number of nodes to service that load and still decrease total power consumption. This is shown by a simple example and the data in Table 2. If the service requirement states the desired throughput is 36000 connections in 30 seconds (with a client request timeout of 5 seconds), 5 cluster nodes as configured in our architecture meet this requirement running in gear 4 . The subsequent total power usage is approximately $5 \cdot 97.66=488$ watts. Four similarly configured servers (without managing the power proactively) could also service this same load; however, the total power usage is $4 \cdot 136.63=547$ watts. This example illustrates a decrease of about $11 \%$ in total power needed. Of the 10 benchmarks created, 9 exhibit the general trend depicted in Figure 3.

The preceding example used an increase in nodes to show the benefit in conforming to a lower power limit. In addition, this same data is used in Table 3 to reflect additional gains in throughput. Notice the gain in throughput at gear 3 is nearly $13 \%$ despite the 70.25 Watts of unused power. Without a more proactive means of power allocation (i.e., an LPA) this represents a loss in throughput.

The preceeding examples help motivate our research but do not reflect the strict representation of all applications. For instance, an exception to the high difference of power and throughput at gear 0 was exhibited in one of our synthentic benchmarks. 


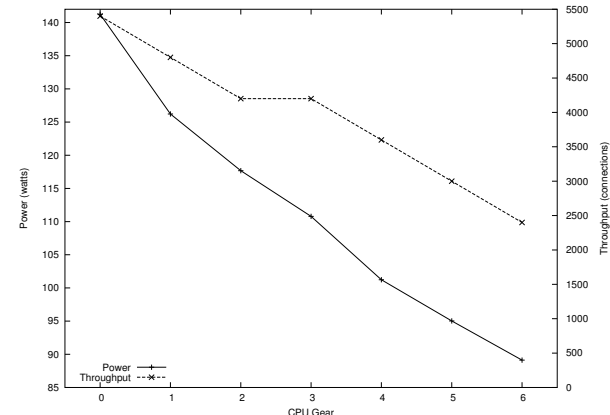

Fig. 4. Prime numbers under 10000 power vs. Fig. 5. Relationship of power and concurrency to performance trade-off.

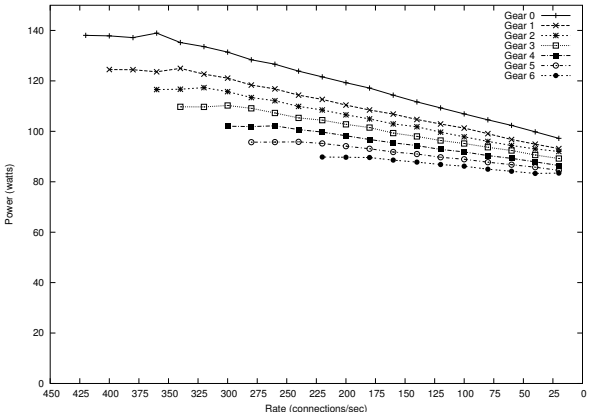

print environment variables.

The resulting power-throughput relationship in Table 4 shows an even larger gain in throughput at lower power gears (i.e., 6 to 5) than the preceeding example. On the other hand, the benefit of the reduced power gear 1 (from 0 ) is not as pronounced. ${ }^{1}$

Another important result emerged from the affects of one benchmark. Figure 4 reflects no gain in throughput going from gear 3 to 2. Unfortunately, there is a $6.21 \%$ increase in power usage for this transition. The data reveals the lower bound overload threshold was just exceeded for gear 3 and the upper bound prevented gear 2 from additional gains. With this result, a power management policy should select the lower power gear.

Rather than just considering total throughput in a given time interval, one can also analyze the power needed to sustain a desirable concurrency. Figure 5 shows an example benchmark illustrating power needed in all seven gears. Depicting the highest performance on the left, in the slowest gear there is a $27.3 \%$ increase in concurrency to go to the next highest gear with a corresponding increase of $6.5 \%$ in power usage. This is noted by comparing the leftmost points of the bottom two curves. At higher performance gears the additional gains in concurrency are perhaps not worth the additional power consumption. The concurrency difference is only $5 \%$ transitioning from gear 1 to 0 with an increase of $10.9 \%$ in power consumption. An opportunity cost analysis such as this can be used in a policy controller that attempts to meet a given level of concurrency.

To verify the efficacy of the LPA, the merge sort CGI is used as a representative benchmark. In the first data set, a 1500 Watt limit is established and extrapolation starts with 10 nodes. This allows an interpolated point to occur between each static gear assignment. In the second data set, a 550 Watt limit is used and extrapolation starts at 3 nodes. To reduce variability and ensure an adequate length of time for the test, throughput measurements were obtained after sustained load was applied for $60 \mathrm{sec}-$ onds. Throughput was again considered maximized based on a 5 second timeout using 4 cluster nodes as request clients. Table 5 depicts the results of the static assignment. Total effective throughput is found by subtracting the number of errors from the total

\footnotetext{
${ }^{1}$ Sort-based benchmarks used the same quantity and distribution of items in all algorithms.
} 
Table 5. Fixed gear throughput, aggregate power, and ideal node power usage $\left(P_{\text {gear }}=\right.$ $\left.P_{\text {global }} / N\right)$.

\begin{tabular}{|r|r|r|r|r|}
\hline$N$ & Gear & Throughput & Power $(\mathrm{W})$ & $P_{\text {gear }}(\mathrm{W})$ \\
\hline \hline 3 & 0 & 62745 & 425.43 & 183.33 \\
\hline 4 & 1 & 77184 & 507.92 & 137.50 \\
\hline 4 & 2 & 71728 & 476.96 & 137.50 \\
\hline 4 & 3 & 64052 & 444.96 & 137.50 \\
\hline 5 & 4 & 71895 & 514.40 & 110.00 \\
\hline 5 & 5 & 57805 & 480.10 & 110.00 \\
\hline 6 & 6 & 53652 & 541.38 & 91.67 \\
\hline \hline 10 & 0 & 209150 & 1418.10 & 150.00 \\
\hline 11 & 1 & 212256 & 1396.78 & 136.36 \\
\hline 12 & 2 & 215184 & 1464.32 & 125.00 \\
\hline 13 & 3 & 208169 & 1446.12 & 115.38 \\
\hline 14 & 4 & 201306 & 1440.32 & 107.14 \\
\hline 15 & 5 & 173415 & 1440.30 & 100.00 \\
\hline 16 & 6 & 143072 & 1443.68 & 93.75 \\
\hline
\end{tabular}

Table 6. Dynamic gear throughput and aggregate power using the LPA $\left(\omega=P_{\text {gear }}\right)$.

\begin{tabular}{|r|r|r|r|}
\hline$N$ & Throughput & Power $(\mathrm{W})$ & $\omega(\mathrm{W})$ \\
\hline \hline 4 & 81600 & 546.96 & 137.50 \\
\hline 5 & 81745 & 547.70 & 110.00 \\
\hline 6 & 63204 & 544.86 & 91.67 \\
\hline \hline 11 & 224103 & 1498.09 & 136.36 \\
\hline 12 & 229584 & 1498.68 & 125.00 \\
\hline 13 & 216112 & 1464.32 & 115.38 \\
\hline 14 & 210826 & 1477.84 & 107.14 \\
\hline 15 & 182700 & 1500.00 & 100.00 \\
\hline 16 & 141232 & 1478.88 & 93.75 \\
\hline
\end{tabular}

connections. Based on the number of nodes and the ideal power limit, the interpolated results using the LPA are shown in Table 6.

Figures 6 and 7 illustrate the benefit of overprovisioning using the data from Tables 5 and 6 . There are two lines depicting throughput, one for an individual node and the other for extrapolated aggregate values. As expected, the individual throughput decreases as available power is reduced. The aggregate throughput curve shows the performance of a cluster. When the power decrease crosses a vertical bar representing the ideal power usage, another node is supported within the power limit. It is important to note that the highest cluster throughput is not the best performance state in both figures.

As the raw data in Tables 5 and 6 show, there is a $7.15 \%$ gain in throughput between gears 0 and 1 for 11 nodes. In addition, there is a $6.69 \%$ gain in throughput between a staticly assigned gear 2 and the LPA using 12 nodes. Notice that after gear 2, there is no resultant benefit from increasing the number of nodes. Additional consideration is warranted if considering an environment, subject to this same limit, that does no proactive management of performance gears. In such a case, only 10 nodes can exist under the limit so the resulting throughput benefit of the LPA using 12 nodes is $9.77 \%$. Clearly these benefits are dependent on the power capacity available and the number of nodes. If starting with 3 nodes, the benefit from a statically assigned solution is approximately $5.91 \%$ using 4 nodes at gear 1 ; moreover, the gain from an unmanaged 3 node solution is $30.28 \%$ with a cluster of 5 LPA managed nodes.

\section{Related Work}

The case for a closer relationship between the operating system and power management is explored in $[9,10]$. Flinn and Satyanarayanan $[11,12]$ show that coordination with 


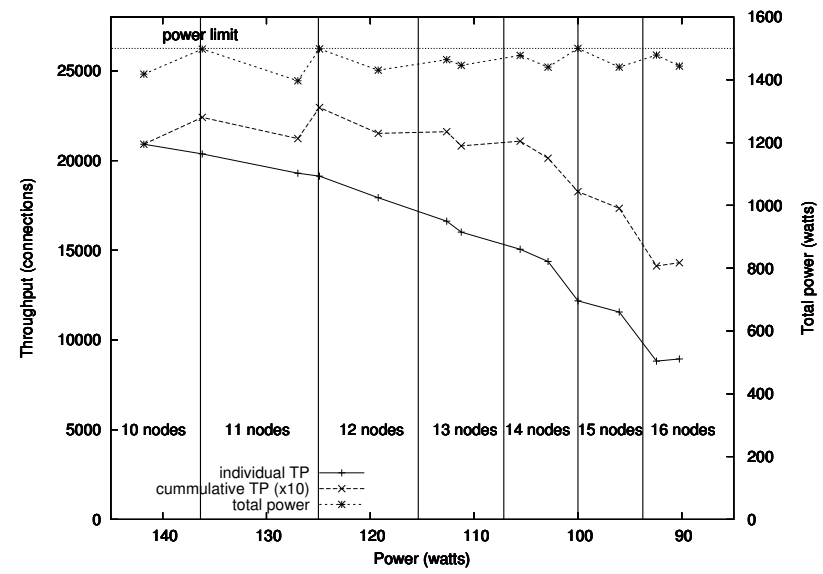

Fig. 6. LPA performance with target power set to $P_{\text {gear }}$ using 1500 Watts starting with 10 nodes.

applications can yield significant power savings. Dynamic voltage scaling (changing both frequency and voltage) to reduce power consumption was explored in [13-17].

A vast amount of research has been done with regards to energy conservation (EC) on mobile platforms [18-22]. The role of EC is complimentary to our goal of efficient power allocation. Power represents the instantaneous energy used at a specific point in time and energy is the usage of power in a defined interval. Our strategy maximizes throughput constrained by the limit using a target power. This power target could be derived from an EC policy; however, it is not a necessary condition.

Power management in commercial servers is important for web servers [23, 24]. Much of this work relies on load balancers to distribute work. An investigation of load balancing was done in $[25,26]$ to turn cluster nodes on or off based on load. Additional research has also been done by Elnozahy et al. [27] for developing mechanisms for energy-efficient clusters using combinations of IVS, CVS, and VOVO policies. Although the VOVO policy is not considered in our initial implementation, its importance is less significant in a mixed architecture environment. In [28], an economic approach is chosen to determine the minimal number of servers required to handle load. Unlike [28] we favor a decentralized approach and seek to maximize throughput. In [29], Sharma et $a l$. applies real-time techniques to web servers in order to conserve energy and maintain QoS. Managing to service metrics is one instance of a target power allocation mechanism in our local controller.

In server farms, disk energy consumption is also important. One study of four energy conservation schemes concludes by stating that reducing spindle speed is the only option for clusters [30]. DRPM is a scheme to modulate the speed of the disk dynamically to save energy $[31,32]$ rather than stopping disk rotation. We plan to investigate this approach in our framework in future efforts.

While analyzing energy efficiency and operating points in [33], it is found that the most energy efficient gear is not always the lower performance point with their concept of critical power slope. Research such as in [34] focus on uniform workload distribution 


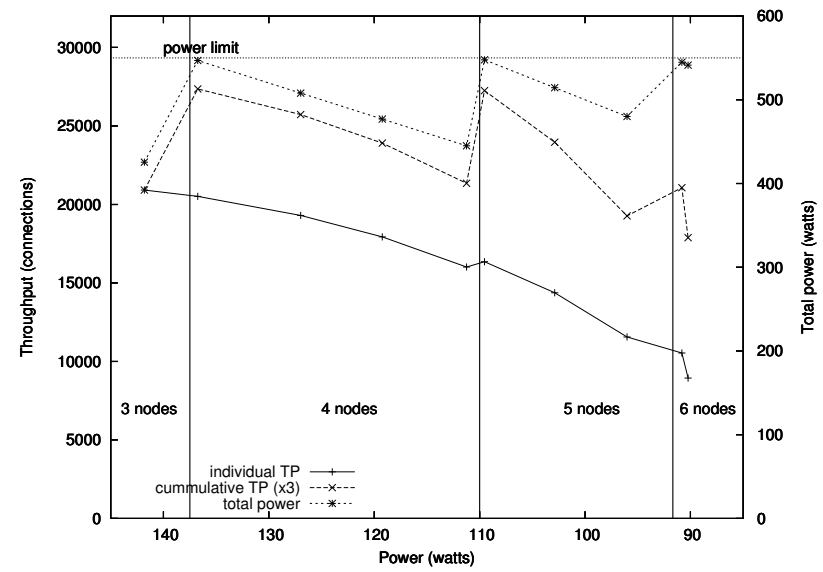

Fig. 7. LPA performance with target power set to $P_{\text {gear }}$ using 550 Watts starting with 3 nodes.

with migratable loads. This work has a contribution towards policy development in the management of power limits. The approach of estimating power consumption using performance counters is taken in [35-37] and is complimentary to our notion of target power assignment.

\section{Future Work}

This paper has investigated the potential of overprovisioning in data centers with a focus on increasing throughput based on a defined power limit. We have presented the preliminary framework used for our research but it only represents an initial foray into the realm of distributed and local power control. Consistent with our approach to make the overall local and global mechanisms perform in a "hands free" operation, we are investigating adjustments to our local controller that will tune it automatically based on the demands of individual devices. Additional work is also needed with regards to optimizing local and global target power policies. Although target power usage has been explored, research on regarding the power limit as an unsurpassable barrier is underway along with providing intra-node differentiated service.

The status of our GPA is constrained due to a limited system for power measurement. A robust implementation is planned to enable future emphasis on nonuniform workloads and heterogeneous architectures. Greater focus and future efforts will also address current shortfalls with regards to initial node power on, synchronization of broadcast data and timing irregularities, and simultaneous cluster node adjustments. The latter will enable us to maintain a tighter upper bound on the true global power limit. The Power Exchange Protocol will also be enhanced with additional capabilities to request or deliver power to any node in the cluster within a transactional framework. This is planned for the direct reallocation of power to handle predictive power conditions not accounted for in the global allocation strategy. 
We have presented a framework to handle safe overprovisioning based on power limits. We have motivated the objective and goals for our research based on preliminary data collected. The additional throughput gains possible from a strategy to manage the power limit can help increase the computational effectiveness of data centers without causing difficulties with existing power infrastructure.

\section{References}

1. Adiga et al., N.: An overview of the BlueGene/L supercomputer. In: Supercomputing 2002. (2002)

2. http://www.acpi.info: Advanced Configuration and Power Interface Specification, Revision 3.0. Hewlett-Packard Corporation, Intel Corporation, Microsoft Corporation, Phoenix Technologies Ltd., and Toshiba Corporation (2004)

3. Mudge, T.: Power: A first class architectural design constraint. IEEE Computer 34 (2001) $52-57$

4. American Power Conversion Corp.: APC power distribution products. http://www . apc.com/products / (2004)

5. Advanced Micro Devices, Inc.: AMD Athlon 64 processor data sheet http: //www.amd.com/us-en/assets/content_type/white_papers_and_ tech_docs\%/24659.PDF (2004)

6. Minerick, R.J., Freeh, V.W., Kogge, P.M.: Dynamic power management using feedback. In: Workshop on Compilers and Operating Systems for Low Power, Charlottesville, Va (2002) 6-1-6-10

7. University of Illinois at Urbana Champaign: The common gateway interface. http:// hoohoo.ncsa.uiuc.edu/cgi/ (2004)

8. Mosberger, D., Jin, T.: httperf: A tool for measuring web server performance. In: WISP, Madison, WI (1998) 59-67

9. Vahdat, A., Lebeck, A., Ellis, C.: Every joule is precious: The case for revisiting operating system design for energy efficiency. In: Proceedings of the 9th workshop on ACM SIGOPS European workshop. (2000) 31-36

10. Ellis, C.: The case for higher-level power management. Proceedings of the 7th Workshop on Hot Topics in Operating Systems (1999)

11. Flinn, J., Satyanarayanan, M.: Energy-aware adaptation for mobile applications. In: Symposium on Operating Systems Principles. (1999) 48-63

12. Flinn, J., Satyanarayanan, M.: Powerscope: A tool for profiling the energy usage of mobile applications. In: Proceedings of the Second IEEE Workshop on Mobile Computing Systems and Applications. (1999)

13. Flautner, K., Reinhardt, S., Mudge, T.: Automatic performance-setting for dynamic voltage scaling. In: Proceedings of the 7th Conference on Mobile Computing and Networking MOBICOM '01. (2001)

14. Gruian, F.: Hard real-time scheduling for low-energy using stochastic data and DVS processors. In: Proceedings of the International Symposium on Low-Power Electronics and Design ISPLED '01. (2001)

15. Pering, T., Burd, T., Brodersen, R.: The simulation and evaluation of dynamic voltage scaling algorithms. In: ISLPED 1998. (1998)

16. Pouwelse, J., LangenDoen, K., Sips, H.: Energy priority scheduling for variable voltage processors. In: Proceedings of the International Symposium on Low-Power Electronics and Design ISPLED '01. (2001) 
17. Im, C., Kim, H., Ha, S.: Dynamic voltage scheduling technique for low-power multimedia applications using buffers. In: Proceedings of the International Symposium on Low-Power Electronics and Design ISPLED '01. (2001)

18. Zeng, H., Ellis, C.S., Lebeck, A.R., Vahdat, A.: Currentcy: Unifying policies for resource management. In: USENIX 2003 Annual Technical Conference. (2003)

19. Anand, M., Nightingale, E., Flinn, J.: Self-tuning wireless network power management. In: Mobicom. (2003)

20. Helmbold, D.P., Long, D.D.E., Sherrod, B.: A dynamic disk spin-down technique for mobile computing. In: Mobile Computing and Networking. (1996) 130-142

21. Douglis, F., Krishnan, P., Bershad, B.: Adaptive disk spin-down policies for mobile computers. In: Proc. 2nd USENIX Symp. on Mobile and Location-Independent Computing. (1995)

22. Li, K., Kumpf, R., Horton, P., Anderson, T.E.: A quantitative analysis of disk drive power management in portable computers. In: USENIX Winter. (1994) 279-291

23. Bohrer, P., Elnozahy, E., Keller, T., Kistler, M., Lefurgy, C., McDowell, C., Rajamony, R.: The case of power management in web servers. In Graybill, R., Melham, R., eds.: Power Aware Computing. Kluwer/Plenum (2002)

24. Lefurgy, C., Rajamani, K., Rawson, F., Felter, W., Kistler, M., Keller, T.W.: Energy management for commerical servers. IEEE Computer (2003) 39-48

25. Pinheiro, E., Bianchini, R., Carrera, E., Heath, T.: Load balancing and unbalancing for power and performance in cluster-based systems. In: Proceedings of the Workshop on Compilers and Operating Systems. (2001)

26. Pinheiro, E., Bianchini, R., Carrera, E.V., Heath, T.: Dynamic cluster reconfiguration for power and performance. In: Compilers and Operating Systems for Low Power. (2001)

27. Elnozahy, E.M., Kistler, M., Rajamony, R.: Energy-efficient server clusters. In: Workshop on Mobile Computing Systems and Applications. (2002)

28. Chase, J.S., Anderson, D.C., Thakar, P.N., Vahdat, A., Doyle, R.P.: Managing energy and server resources in hosting centers. In: Symposium on Operating Systems Principles. (2001) 103-116

29. Sharma, V., Thomas, A., Abdelzaher, T., Skadron, K.: Power-aware QoS management in web servers. In: 24th Annual IEEE Real-Time Systems Symposium, Cancun, Mexico (2003)

30. Carrera, E.V., Pinheiro, E., Bianchini, R.: Conserving disk energy in network servers. In: Proceedings of International Conference on Supercomputing, San Fransisco, CA (2003) 8697

31. Gurumurthi, S., Sivasubramaniam, A., Kandemir, M., Franke, H.: Dynamic speed control for power management in server class disks. In: Proceedings of International Symposium on Computer Architecture. (2003) 169-179

32. Gurumurthi, S., Sivasubramaniam, A., Kandemir, M., Franke, H.: Reducing disk power consumption in servers with DRPM. IEEE Computer (2003) 41-48

33. Miyoshi, A., Lefurgy, C., Hensbergen, E.V., Rajamony, R., Rajkumar, R.: Critical power slope: Understanding the runtime effects of frequency scaling. In: Proceedings of the 16th International Conference on Supercomputing. (2002) 35-44

34. Bradley, D., Harper, R., Hunter, S.: Workload-based power management for parallel computer systems. IBM Journal of Research and Development 47 (2003) 703-718

35. Bellosa, F.: The benefits of event-driven energy accounting in power-sensitive systems. In: Proceedings of the 9th ACM SIGOPS European Workshop. (2000)

36. Joseph, R., Martonosi, M.: Run-time power estimation in high performance microprocessors. In: Proceedings of the International Symposium on Low-Power Electronics and Design ISPLED '01. (2001)

37. Gniady, C., Hu, Y.C., Lu, Y.H.: Program counter based techniques for dynamic power management. In: Proceedings of the 10th International Symposium on High-Performance Computer Architecture. (2004) 